\title{
Downregulation of TNFAIP2 suppresses proliferation and metastasis in esophageal squamous cell carcinoma through activation of the Wnt/ß-catenin signaling pathway
}

\author{
YUNBO XIE ${ }^{1,2}$ and BIN WANG ${ }^{1}$ \\ ${ }^{1}$ Department of Cardiothoracic Surgery and ${ }^{2}$ Chongqing Key Laboratory of Molecular Oncology and Epigenetics, \\ The First Affiliated Hospital of Chongqing Medical University, Chongqing 400016, P.R. China
}

Received August 10, 2016; Accepted September 20, 2016

DOI: $10.3892 /$ or.2017.5557

\begin{abstract}
Tumor necrosis factor- $\alpha$ (TNF- $\alpha$ ) plays a pivotal role in malignant tumor formation in the tumor microenvironment. To investigate the role of TNF- $\alpha$ in esophageal squamous cell carcinoma (ESCC), we assessed expression profiles of the downstream gene TNF- $\alpha$-induced protein 2 (TNFAIP2), which e previously unknown in ESCC. TNFAIP2 mRNA and protein expression levels were examined by qRT-PCR and immunohistochemical analysis in 24 fresh and 55 paraffin-embedded specimens, respectively. The results demonstrated that TNFAIP2 mRNA and protein levels were overexpressed in tumor cells, and TNFAIP2 overexpression was significantly associated with $\mathrm{T}$ stage $(\mathrm{p}=0.049), \mathrm{N}$ stage $(\mathrm{p}=0.019)$ and the International Union Against Cancer (UICC) stage $(\mathrm{p}=0.028)$. In vitro, TNFAIP2 was highly expressed in TNF $\alpha$-stimulated Eca109, Kyse150, Kyse510 and TE-10 cells. Lentivirus-mediated RNA interference of TNFAIP2 inhibited cell proliferation, colony formation, migration, invasion and the cell cycle. Moreover, LV-RNAi-mediated TNFAIP2 was found to regulate the $\mathrm{Wnt} / \beta$-catenin by decreasing expression of some genes downstream from $\beta$-catenin (i.e., C-myc, cyclin D1, MMP-7 and Snail), and upregulating expression of E-cadherin and p-GSK-3 $\beta$. Taken together, these results show that TNFAIP2 may be a potential tumorigenesis gene in ESCC. Our data indicate that TNFAIP2 overexpression may facilitate proliferation and metastasis via activation of the Wnt $/ \beta$-catenin signaling pathway in ESCC.
\end{abstract}

\section{Introduction}

The majority (90\%) of cases of esophageal cancer in China are esophageal squamous cell carcinomas (ESCC). The high

Correspondence to: Professor Bin Wang, Department of Cardiothoracic Surgery, The First Affiliated Hospital of Chongqing Medical University, 1 Youyi Road, Yuzhong, Chongqing 400016, P.R. China

E-mail: 18908399668@189.cn

Key words: TNFAIP2, cell apoptosis, cell invasion, cell migration, tumorigenesis incidence of ESCC in China is part of the aptly named 'Asian Esophageal Cancer Belt', which is characterized by a low 5-year survival rate and high incidence and mortality rates, although, contributing risk factors and mechanisms of ESCC remain unknown $(1,2)$.

Tumor necrosis factor- $\alpha$ (TNF- $\alpha)$ is a key cytokine involved in the onset of chronic inflammation, which plays a major role in the tumor microenvironment. There is a vicious cycle between tumor formation and inflammation, in which inflammation promotes tumor formation, and tumor formation promotes inflammation (3). TNF $\alpha$ is a major mediator of tumor-related inflammation in the tumor microenvironment and abundant evidence has demonstrated that TNF $\alpha$ promotes tumor growth, metastatic and invasive activities, and stimulation of neoangiogenesis in the tumor microenvironment in breast, ovarian, colorectal, prostate, bladder and renal cancers, as well as melanoma and esophageal carcinoma (3-8). However, the mechanisms through which TNFa promotes tumor progression remain unknown.

TNF- $\alpha$-induced protein 2 (TNFAIP2) is located on chromosome 14q32 and encodes a protein consisting of 654 amino acids. TNFAIP2 is a member of the Sec6 family and was originally identified as a TNF- $\alpha$-induced protein in human endothelial cells (9). Rusiniak et al found that TNFAIP2 is a potential target for transcriptional repression by the acute promyelocytic leukemia (PML)-retinoic acid receptor $\alpha$ fusion protein (10). Moreover, TNFAIP2, as an independent prognostic indicator in nasopharyngeal carcinoma, is highly expressed in nasopharyngeal carcinoma, and promotes migration and invasion likely by modulating actin organization $(11,12)$.

Based on these pivotal findings, we hypothesized that TNFAIP2 plays a role in the tumorigenesis and metastasis of ESCC and thus is a potential therapeutic target for ESCC. However, there are relatively few studies of the molecular functions of TNFAIP2, and the possible associations with tumorigenesis and metastasis in ESCC remain unclear.

\section{Materials and methods}

Patients and clinical specimens. A total of 79 ESCC specimens (55 paraffin-embedded and 24 fresh) with adjacent 
free-cancerous esophageal tissues that were frozen and stored in liquid nitrogen were histopathologically diagnosed at the Department of Pathology of Chongqing Medical University (Chongqing, China), and the Department of Cardiothoracic Surgery of The First Affiliated Hospital of Chongqing Medical University (Chongqing, China), respectively, from 2013 to 2015. Clinical and tumor (T)-lymph node (N)-metastasis (M) staging was performed in accordance with the guidelines of the 6th edition of the International Union Against Cancer (UICC). None of the patients received radiation or chemotherapy before surgery. Clinical patient information is summarized in Table I. The study protocol was approved by the Institutional Research Ethics Committee of The First Affiliated Hospital of Chongqing Medical University and informed consent was obtained from all patients.

Immunohistochemical analysis. Immunohistochemical analysis of 55 human paraffin-embedded ESCC samples was performed to study the expression profiles of TNFAIP2, $\beta$-catenin and E-cadherin using anti-human TNFAIP2 antibody (dilution, 1:100; cat. no. sc-30138; Santa Cruz Biotechnology, Inc., Santa Cruz, CA, USA), $\beta$-catenin antibody (dilution, 1:100; cat. no. 8480) and E-cadherin antibody (dilution, 1:400; cat. no. 3195) (both from Cell Signaling Technology, Inc., Danvers, MA, USA). The procedure was similar to previously described methods (11,13-15). The histopathological features and patient data of the samples were evaluated via the $\mathrm{H}$-scores method by two board-certified pathologists who were blinded to patient data. The intensity of specific staining was defined as negative (0), weakly positive (1+), somewhat positive (2+), strongly positive (3+) and very strongly positive (4+). H-score $=\Sigma P_{i}$ $(i+1)$, where $P_{i}$ is the percentage of stained cells in each intensity category; $i=1-4$. Total scores $\geq 300$ were characterized as a high-level expression $(16,17)$.

Western blot analysis. Protein extraction and western blot analysis were performed as previously described $(15,18)$. Briefly, the cells were lysed with lysis buffer containing protease inhibitors and the lysates ( $40 \mu \mathrm{g} /$ lane) were separated by sodium dodecyl sulfate-polyacrylamide gel electrophoresis (SDS-PAGE). Then, proteins were transferred to a polyvinylidene fluoride membrane, which was incubated with appropriate secondary antibodies [i.e., anti-TNFAIP2 (dilution, 1:1,000; cat. no. sc-30138; Santa Cruz Biotechnology, Inc.), $\beta$-catenin (1:1,000; cat. no. 8480), E-cadherin (dilution, 1:1,000; cat. no. 3195; both from Cell Signaling Technology, Inc.), c-myc (dilution, 1:1,000; cat. no. ab32072; Abcam, Cambridge, UK), cyclin D1 (dilution, 1:1,000; cat. no. 2978), MMP-7 (dilution, 1:1,000; cat. no. 3801), p-GSK-3 $\beta$ (dilution, 1:1,000; cat. no. 9322), GSK-3 $\beta$ (1:1,000; cat. no. 12456), Snail (dilution, 1:1,000; cat. no. 3879) (all from Cell Signaling Technology, Inc.), and $\beta$-actin, respectively] that were detected and visualized using an enhanced chemiluminescence detection system (BeyoECL Plus; Beyotime Institute of Biotechnology, Shanghai, China).

Cell culture. Eca109 and Kyse150 cells, both ESCC cell lines, were maintained in Roswell Park Memorial Institute (RPMI)-1640 medium (Corning, Inc., Corning, NY, USA) supplemented with $10 \%$ fetal bovine serum (FBS), $100 \mathrm{U} / \mathrm{ml}$
Table I. Relationships between TNFAIP2 expression and clinicopathological features of the ESCC patients.

\begin{tabular}{|c|c|c|c|c|}
\hline Characteristics & Total (n) & High & Low & P-value \\
\hline Age (years) & & & & 0.66 \\
\hline$\geq 63^{\mathrm{b}}$ & 32 & 19 & 13 & \\
\hline$<63$ & 23 & 15 & 8 & \\
\hline Gender & & & & 0.288 \\
\hline Male & 43 & 25 & 18 & \\
\hline Female & 12 & 9 & 3 & \\
\hline Differentiation & & & & 0.408 \\
\hline G1 & 16 & 8 & 8 & \\
\hline $\mathrm{G} 2$ & 24 & 17 & 7 & \\
\hline G3 & 15 & 9 & 6 & \\
\hline T stage & & & & $0.041^{\mathrm{a}}$ \\
\hline $\mathrm{T} 1$ & 8 & 2 & 6 & \\
\hline $\mathrm{T} 2$ & 9 & 4 & 5 & \\
\hline $\mathrm{T} 3$ & 28 & 20 & 8 & \\
\hline $\mathrm{T} 4$ & 10 & 8 & 2 & \\
\hline $\mathrm{N}$ stage & & & & $0.019^{\mathrm{a}}$ \\
\hline No & 28 & 13 & 15 & \\
\hline N1 & 14 & 9 & 5 & \\
\hline N2 & 13 & 12 & 1 & \\
\hline UICC stage & & & & $0.023^{\mathrm{a}}$ \\
\hline I & 11 & 3 & 8 & \\
\hline II & 23 & 15 & 8 & \\
\hline III & 21 & 16 & 5 & \\
\hline$\beta$-catenin & & & & $0.006^{\mathrm{a}}$ \\
\hline High & 36 & 27 & 9 & \\
\hline Low & 19 & 7 & 12 & \\
\hline E-cadherin & & & & $<0.001$ \\
\hline High & 18 & 5 & 13 & \\
\hline Low & 37 & 29 & 8 & \\
\hline
\end{tabular}

${ }^{a}$ With statistical significance, $\mathrm{p}$-value $<0.05$; ${ }^{\mathrm{b}}$ median age $=63$ years. UICC, International Union Against Cancer.

of penicillin, and $100 \mu \mathrm{g} / \mathrm{ml}$ of streptomycin, and incubated in a humidified atmosphere of $5 \% \mathrm{CO}_{2}$ at $37^{\circ} \mathrm{C}$.

Treatment of cells with recombinant human (rh)-TNF $\alpha$. rh-TNFa (cat. no. 10602; Sino Biological, Inc., Beijing, China) was diluted with complete medium to a concentration of $25 \mathrm{ng} / \mathrm{ml}$. Cells were treated with rh-TNF $\alpha$ for $48 \mathrm{~h}$ and TNFAIP2 mRNA and protein expression levels were detected by quantitative real-time PCR (qRT-PCR) and western blot analysis, respectively. All experiments were repeated three times. The results of qRT-PCR were normalized with $\beta$-actin using the $2^{-\Delta \Delta C t}$ method (19).

Production of lentiviral constructs and infection. Lentivirus RNA interference of TNFAIP2 (LV-RNAi TNFAIP2 shRNA, 5'-TCTTCACCAAAGGGAAGAA-3'); and a negative control 
lentiviral vector (LV-CON shRNA, 5'-TTCTCCGAACGTGT CACGT-3') were designed and synthesized by GeneChem Co., Ltd. (Shanghai, China). The lentivirus titers were $5 \times 10^{8}$ infectious $\mathrm{U} / \mathrm{ml}$.

To determine the multiplicity of infection (MOI) of the Eca109 and Kyse150 cell lines, a pre-experiment was performed. Briefly, Eca109 and Kyse150 cells ( $2 \times 10^{3}$ cells/well) were cultured in 96-well plates for $12 \mathrm{~h}$, and then infected with $0.2 \mu \mathrm{l}$ (MOI=10), $0.4 \mu \mathrm{l}(\mathrm{MOI}=20)$ or $0.6 \mu \mathrm{l}(\mathrm{MOI}=30)$ of lentivirus diluted with serum-free medium to $1 \times 10^{8}$ infectious $\mathrm{U} / \mathrm{ml}$, respectively. After $12 \mathrm{~h}$, the medium was replaced with fresh complete medium. After three days, 95\% transduction efficiencies of the Eca109 and Kyse150 cells were infected at an MOI of 30 and 20, respectively.

According to the results of the pre-experiment, the Kyse150 and Eca109 cells $\left(2 \times 10^{4}\right.$ cells/well) were cultured in 6-well plates and transfected with 4 and $6 \mu 1$ of the diluted lentivirus, respectively. To obtain stable transfection, puromycin $(1 \mu \mathrm{g} / \mathrm{ml})$ was added to the cultures at $72 \mathrm{~h}$ after transfection. The RNAi effectiveness was validated by qRT-PCR and western blot analysis.

Total RNA extraction, reverse transcription and qRT-PCR. Total RNA was isolated from esophagus carcinoma tissues and Ec109 and Kyse150 cells, using RNAiso Plus Total RNA extraction reagent (cat. no. 9108; Takara Bio, Inc., Otsu, Japan). The PrimeScript ${ }^{\mathrm{TM}}$ RT reagent kit with gDNA Eraser (Perfect Real-Time) (cat. no. RR047A; Takara Bio, Inc.) was used for reverse transcription of total RNA $(1 \mu \mathrm{g})$. qRT-PCR was performed with an Applied Biosystems 7500 Real-Time PCR System (Life Technologies, Carlsbad, CA, USA) using SYBR ${ }^{\circledR}$ Premix Ex Taq ${ }^{\mathrm{TM}}$ II kit (Tli RnaseH Plus) (cat. no. RR820A, Takara Bio, Inc.) according to the manufacturer's instructions. $\beta$-actin was used as an endogenous control to normalize the relative expression level of TNFAIP2. The following primer pairs were used to amplify the mRNA of TNFAIP2 and $\beta$-actin: (F) 5'-CCTGCTCTCCCTACGC-3' and (R) 5'-CGTC CAAGATGCTCCG-3' (11); and (F) 5'-CTCCTCCTGAGC GCAAGTACTC-3' and (R) 5'-TCCTGCTTGCTGATCCACA TC-3 (13), respectively.

Cell migration and invasion assays. Migration and invasion assays were performed as described by Huang et al and Whitington et al $(15,20)$. Briefly, lentivirus-transfected Eca109 and Kyse150 cells were plated at concentrations of $5 \times 10^{4}$ and $1 \times 10^{5}$ cells $/ 8-\mu \mathrm{m}$ Transwell insert with or without Matrigel coating (both from Corning, Inc.) and incubated for $48 \mathrm{~h}$, respectively. Cells that had penetrated the membranes were quantified by counting the number of cells in five random microscopic fields. All experiments were performed in triplicate.

Cell colony formation and proliferation assays. LV-RNAi TNFAIP2 and LV-CON group cells were trypsinized and resuspended at $2 \times 10^{4}$ cells $/ \mathrm{ml}$. Then, $25 \mu \mathrm{l}$ (for colony formation; total volume, $2 \mathrm{ml}$ ) and $100 \mu \mathrm{l}$ (for proliferation) of the cell suspensions were added to each well of 6- and 96-well plates, respectively. Cells plated in the 6-well plates were incubated for 10-14 days and the formed colonies were fixed with $4 \%$ paraformaldehyde and stained with crystal violet for $20 \mathrm{~min}$, respectively. After 24,48 and $72 \mathrm{~h}, 10 \mu \mathrm{l}$ of Cell Counting Kit-8 (CCK-8; reagent) (Dojindo Molecular Technologies, Inc., Tokyo, Japan) was added to each well of the 96-well plates. Cell proliferation was determined using a microplate reader (Infinite 200 PROs; Tecan Trading AG, Männedorf, Switzerland) at designed time points by reading the absorbance at $450 \mathrm{~nm}$ after CCK- 8 treatment for $2 \mathrm{~h}$. Values of proliferation were obtained from five replicate wells for each treatment and time point. Each experiment was performed in triplicate.

Cell cycle and apoptosis analysis. Trypsinized cells were resuspended, washed, centrifuged with phosphate-buffered saline (PBS) at $4^{\circ} \mathrm{C}$ three times, and then fixed with ice-cold $70 \%$ ethanol overnight at $4^{\circ} \mathrm{C}$ for the cell cycle assay or resuspended in 1,000 $\mu 1$ of PBS for the cell apoptosis assay. The cell cycle and apoptosis assays were conducted at Chongqing Medical University Life Sciences Institute. Each assay was independently repeated three times.

Statistical analysis. SPSS 22 statistical software package (SPSS, Inc., Chicago, IL, USA) was used to perform all statistical analyses. The statistical significance of the qRT-PCR results for TNFAIP2 gene expression and for the observations on the basis of cell proliferation, colony formation, migration, invasion, cycle and apoptosis were assessed using Student's $\mathrm{t}$-tests (two-tailed). The Pearson $\chi^{2}$ test was applied to evaluate the relationships between TNFAIP2 expression and expression of $\beta$-catenin and E-cadherin and various clinicopathological characteristics. The Kaplan-Meier method and the log-rank test were used to plot and compare the survival curves, respectively. Data shown are presented as the mean \pm SD or SEM from triplicate experiments as indicated. Significant differences were P-value $<0.05$.

\section{Results}

TNFAIP 2 mRNA and protein overexpression in ESCC clinical tissue specimens. TNFAIP2 was identified as an angiogenic factor and can be induced in umbilical vein endothelial cells by TNF- $\alpha$ (9). Furthermore, TNFAIP2 regulates invasion and metastasis in nasopharyngeal carcinoma (11), and its genetic polymorphism was found to influence ESCC, squamous cell carcinoma of the head and neck and gastric cancer (21-23). However, the mechanisms underlying TNFAIP2 regulation in ESCC remain unknown. Hence, immunohistochemical staining with anti-TNFAIP2 antibody was used to examine TNFAIP2 protein expression in 55 paraffin-embedded ESCC specimens which showed high levels in 34 (62\%) samples and low levels in 21 (38\%) samples, respectively. In contrast, TNFAIP2 was undetectable in the adjacent normal epithelium (Fig. 1A). Moreover, TNFAIP2 mRNA expression was quantified by qRT-PCR, which showed that mRNA levels in $16(67 \%)$ of 24 samples were higher than levels noted in the adjacent normal tissues (Fig. 1B).

Correlations between patient characteristics, clinical features and TNFAIP 2 expression. The patient characteristics and clinical features shown in Table I were evaluated to identify correlations between TNFAIP 2 and clinicopathological 


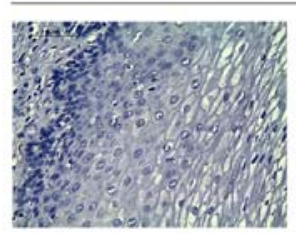

Normal

B

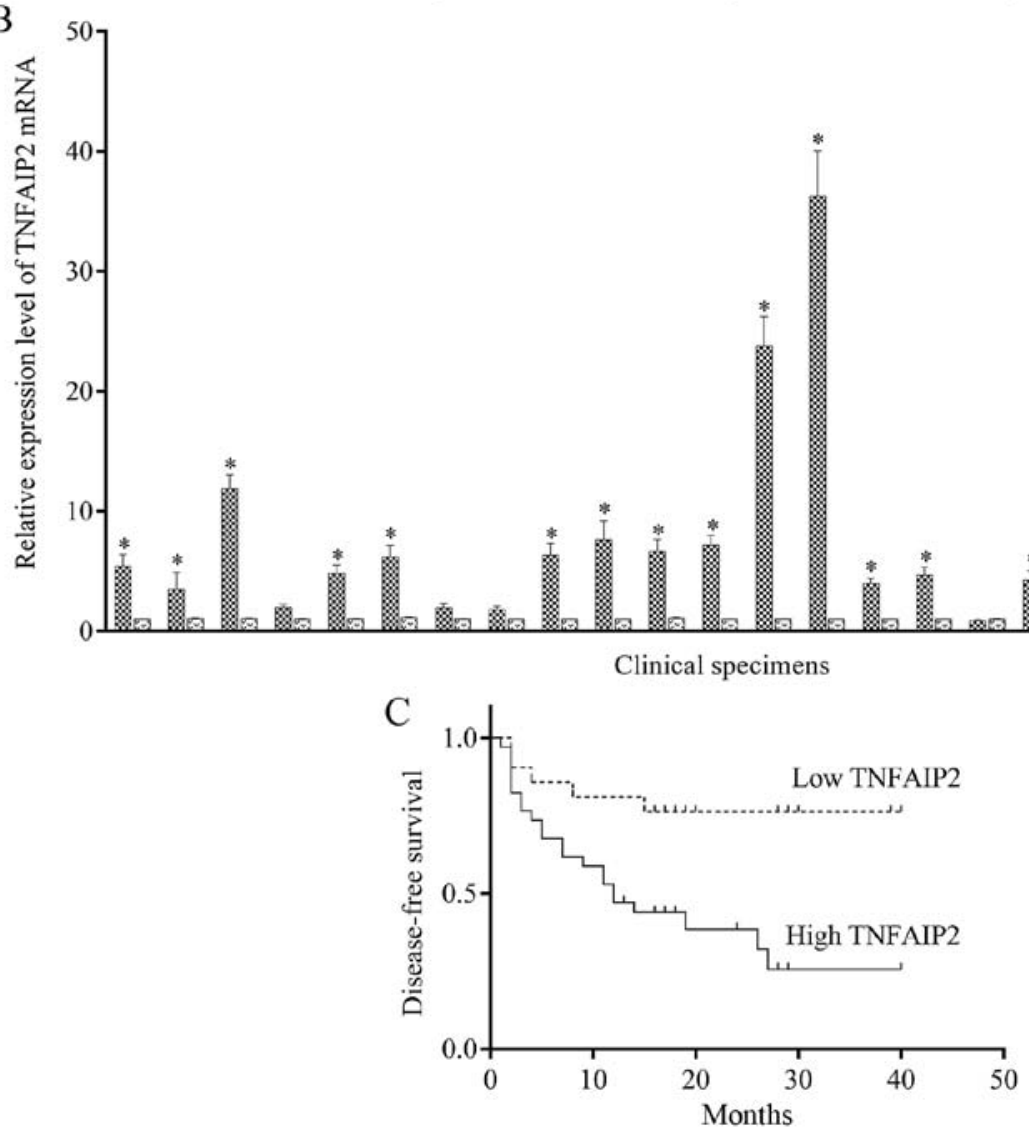

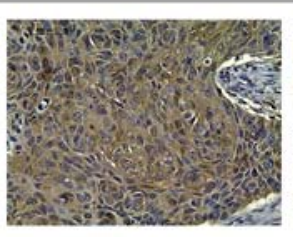

T2 stage

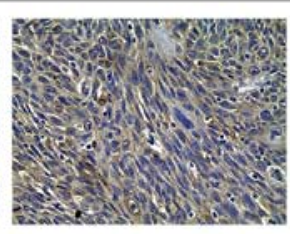

T1 stage

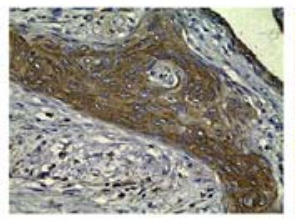

T3 stage

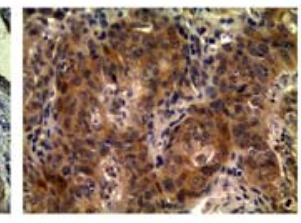

T4 stage

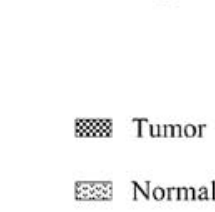

Figure 1. Expression of TNFAIP2 in ESCC samples. (A) The gradual increase in TNFAIP2 expression was associated with T stage (magnification, $\mathrm{x} 40$ ). (B) Relative expression of TNFAIP2 mRNA was examined in 24 ESCC clinical fresh specimens. The data are presented as the mean \pm standard deviation of three independent experiments; ${ }^{*} \mathrm{p}<0.05$. (C) Disease-free survival, using Kaplan-Meier survival analysis, was associated with TNFAIP2 expression in 55 ESCC patients; $\mathrm{p}=0.0057$.

characteristics of ESCC patients, which showed that there were no significant correlations between TNFAIP2 expression and age, gender and cellular differentiation. However, there were significant associations between TNFAIP2 expression and T, N and UICC stage. As shown in Fig. 1A, TNFAIP2 expression gradually increased along with the $\mathrm{T}$ stage.

Association between TNFAIP2 and disease-free survival (DFS). Kaplan-Meier survival analysis and the log-rank test were used to investigate potential impacts of TNFAIP2 expression on the DFS of patients. The median followup period was 20 months and at the time of the present study 28 patients survived and 27 had died. Kaplan-Meier survival curves showed that DFS was obviously higher with low TNFAIP2 expression than high expression (Fig. 1C). Moreover, univariate Cox regression analysis revealed a correlation between DFS and TNFAIP2 expression $(\mathrm{p}=0.023$; regression coefficient $=1.047$; hazard ratio $=2.849$; $95 \%$ confi- dence interval $(\mathrm{CI})=1.152-7.044)$. These results indicate that elevated TNFAIP2 expression may be a risk factor for ESCCassociated mortality.

TNFAIP2 mRNA and protein overexpression in TNF $\alpha$-treated cells. The results of the present study showed that aberrant TNFAIP2 expression in ESCC clinical specimens was correlated with intratumoral T, N and UICC stage. qRT-PCR and western blotting were used to detect THFAIP2 expression levels in Eca109, Eca9706, TE-10, Kyse150 and Kyse510 cells. As shown in Fig. 2A, Eca109 and Kyse150 were used to study their biological functions influenced by TNFAIP2 in vitro. In this experiment, Eca109, Kyse150, Kyse510 and TE-10 cells were treated with $25 \mathrm{ng} / \mathrm{ml}$ of rh-TNF $\alpha$ for $48 \mathrm{~h}$. As shown by the results in Fig. 2B, there were significant differences in TNFAIP 2 mRNA and protein expression levels between treated and untreated cells, indicating that TNFAIP2 can be induced and regulated by TNF $\alpha$. 

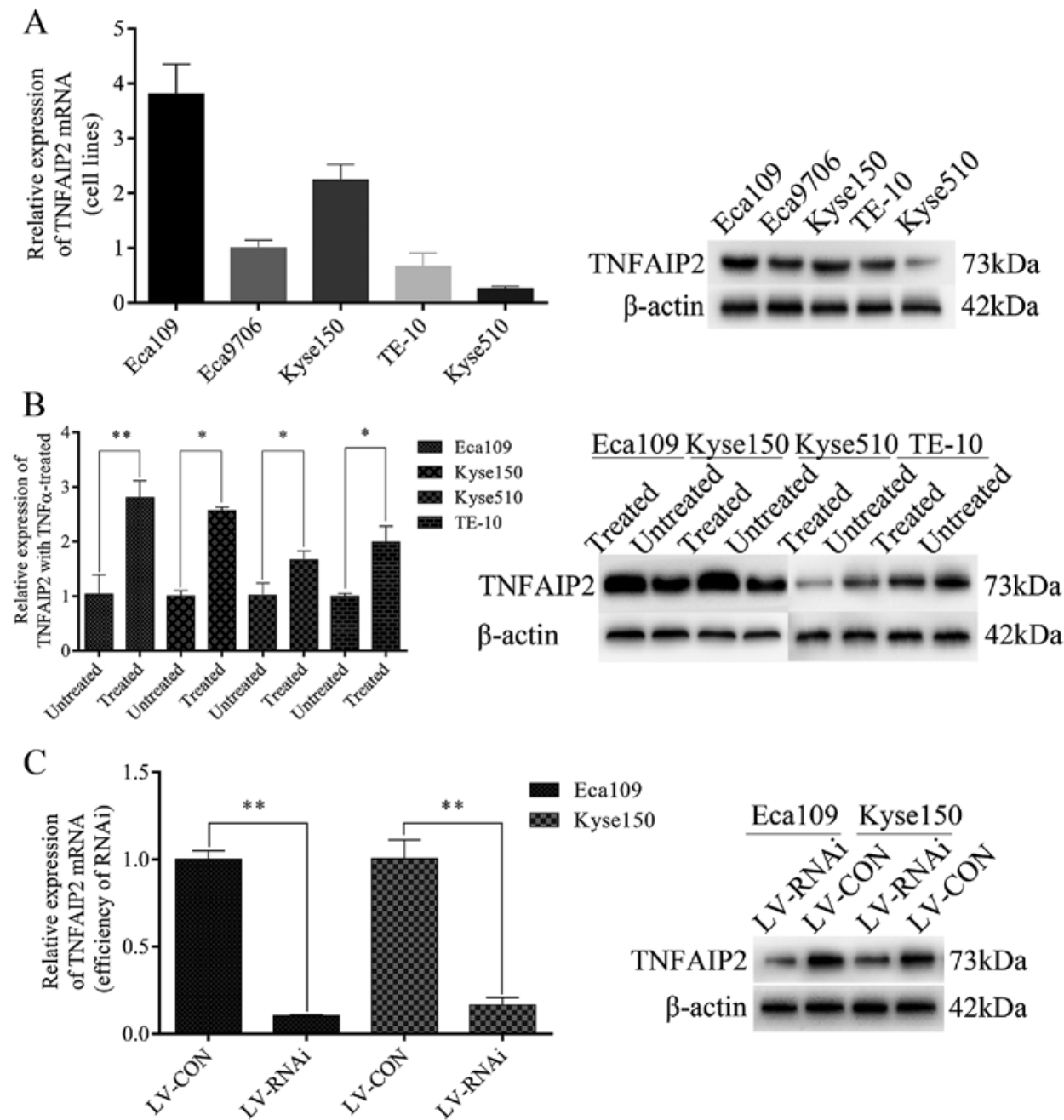

Figure 2. TNFAIP2 expression was examined in ESCC cell lines. (A) Relative expression of TNFAIP2 mRNA in Eca109, Eca9706, Kyse150, Kyse510 and TE-10 cell lines. The relative fold-change of TNFAIP2 expression in five ESCC cell lines was normalized to that of the control cells (Eca9706), which were assigned a value of 1.0. (B) TNF $\alpha$-induced TNFAIP2 mRNA and protein expression levels in Eca109, Kyse150, Kyse510 and TE-10 cells. The experiments were performed in triplicate, and the results are presented as the mean \pm standard deviation; ${ }^{*} \mathrm{p}<0.05,{ }^{* *} \mathrm{p}<0.001$. (C) The efficiency of TNFAIP2 knockdown were $>75 \%$ in mRNA and protein levels. Data are presented as the mean \pm standard deviation; ${ }^{* *} \mathrm{p}<0.001$.

LV-RNAi-mediated TNFAIP2 knockdown decreases tumorigenic and metastatic properties in vitro. Cell colony formation, proliferation, cell cycle, apoptosis, migration and invasive ability play significant roles in tumor tumorigenic and metastatic properties. Therefore, lentiviral RNA interference (LV-RNAi) was used to knock down TNFAIP2 in the ESCC cell lines Eca109 and Kyse150 using techniques to study biological functions of cell lines in vitro with an empty lentiviral vector as a negative control (LV-CON). The efficiencies of knockdown and transfection were $>75$ and $90 \%$ at the mRNA and protein levels (Fig. 2C), respectively.

Colony formation was significantly inhibited in the LV-RNAi group, as compared with the LV-CON group (Fig. 3A1 and A2). LV-RNAi TNFAIP2 resulted in obvious decreased proliferation of the Eca109 and Kyse150 cells compared with the LV-CON group (Fig. 3A3 and A4).

A Transwell assay was employed to examine the role of TNFAIP2 in cellular migration and invasion. As shown by the Transwell assay results presented in Fig. 3B1-B4, cell migration and invasive ability were significantly declined in the LV-RNAi group as compared with the LV-CON group. To investigate potential mechanisms underlying LV-RNAi-induced inhibition of colony formation and proliferation, cell cycle distribution and apoptosis of cells in the LV-RNAi and LV-CON groups were detected by flow cytometry, which showed that in the LV-RNAi group, the Eca109 and Kyse150 cells were mainly arrested in the G0/G1 phase and the percentages of cells in the $\mathrm{G} 2$ and $\mathrm{S}$ phases were decreased (Fig. 3C1-C4), but, there were no significant differences in apoptosis between the LV-RNAi and LV-CON groups ( $\mathrm{p}=0.292)$. Notably, these findings demonstrated that an abundance of TNFAIP2 plays an oncogenic role in ESCC.

Expression of $\beta$-catenin is correlated with TNFAIP2 expression. Several pathways were investigated to elucidate the molecular mechanisms underlying LV-RNAi-mediated inhibition of tumorigenic and metastatic properties. The results showed discrepancies between the interrelated members of the Wnt/ $\beta$-catenin signaling pathway expressed in the LV-RNAi TNFAIP2 group and LV-CON group cells. On the assumption that $\beta$-catenin expression could be upregulated by TNFAIP2, ESCC cell lines stably transfected with lentiviral particles 

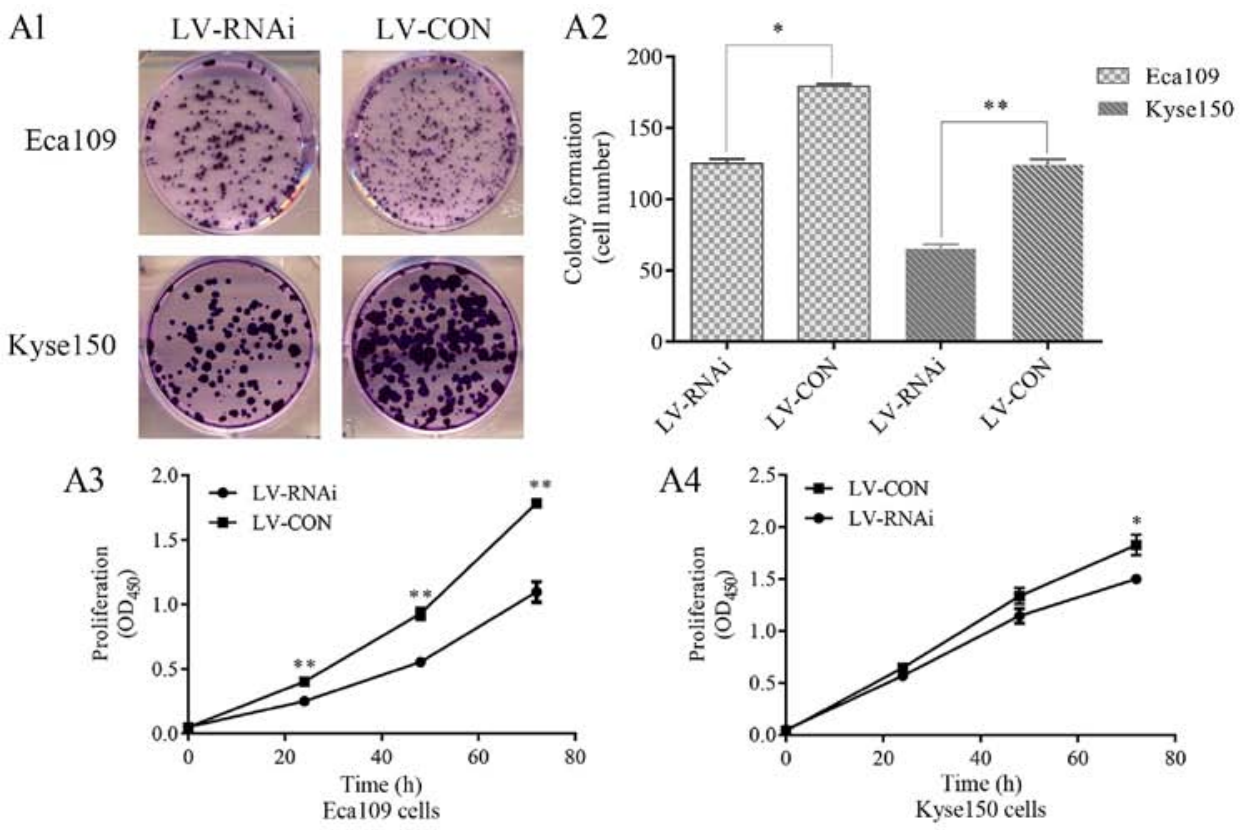

$\mathrm{B} 1$

Cell Migration

B2

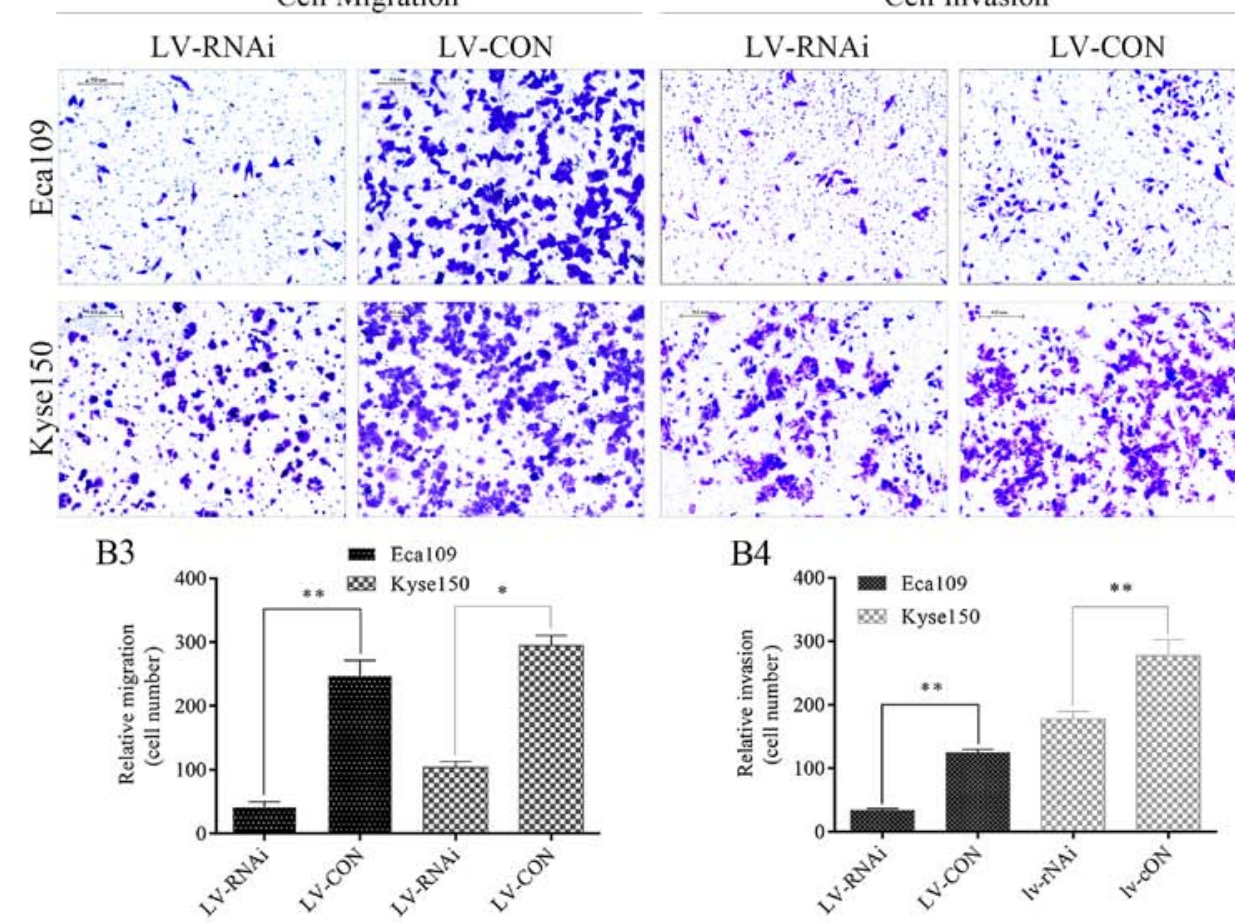

Figure 3. Lentivirus-mediated RNA interference (LV-RNAi) of TNFAIP2 decreased cell colony formation, proliferation, migration and invasion, and arrested the cell cycle in the $\mathrm{G} 0 / \mathrm{G} 1$ phase. (A1-A4) Colony formation (cell number) and cell proliferation $\left(\mathrm{OD}_{450}\right)$ were inhibited in the LV-RNAi group as compared with the LV-CON group. The data from three independent experiments are presented as the mean \pm standard deviation; ${ }^{*} \mathrm{p}<0.05$, ${ }^{* *} \mathrm{p}<0.001$. (B1-B4) Migration and invasion were partially inhibited in the LV-RNAi group. Cells were counted in five random fields with magnifications, of x10. The experiments were performed in triplicate, and the results are presented as the mean \pm standard deviation; ${ }^{*} \mathrm{p}<0.05,{ }^{* *} \mathrm{p}<0.001$.

carrying TNFAIP2 RNAi were used to identify the underlying mechanisms by western blot and qRT-PCR analyses of the cell lysates. Obviously, when TNFAIP2 expression was downregulated, the protein expression levels of $\beta$-catenin were decreased synchronously (Fig. 3D). Moreover, expression levels of p-GSK-3 $\beta$ (upstream of $\beta$-catenin), c-Myc, cyclin D1, matrix metalloproteinase-7 (MMP-7), Snail (downstream) and E-cadherin were evaluated by western blot analysis, which indicated that expression of c-Myc, cyclin D1, MMP-7 and
Snail decreased, while expression levels of E-cadherin and p-GSK-3 $\beta$ were increased in the LV-RNAi group, as compared with the LV-CON group (Fig. 3D).

To elucidate potential associations between TNFAIP2 and $\beta$-catenin, immunohistochemical staining analysis with $\beta$-catenin antibody was conducted using 55 paraffin-embedded specimens. As shown in Fig. 4A, $\beta$-catenin expression (in cytoplasm or nucleus) was relatively high in $36(65 \%)$ samples and relatively low in $19(35 \%)$. $\beta$-catenin expression was found to 

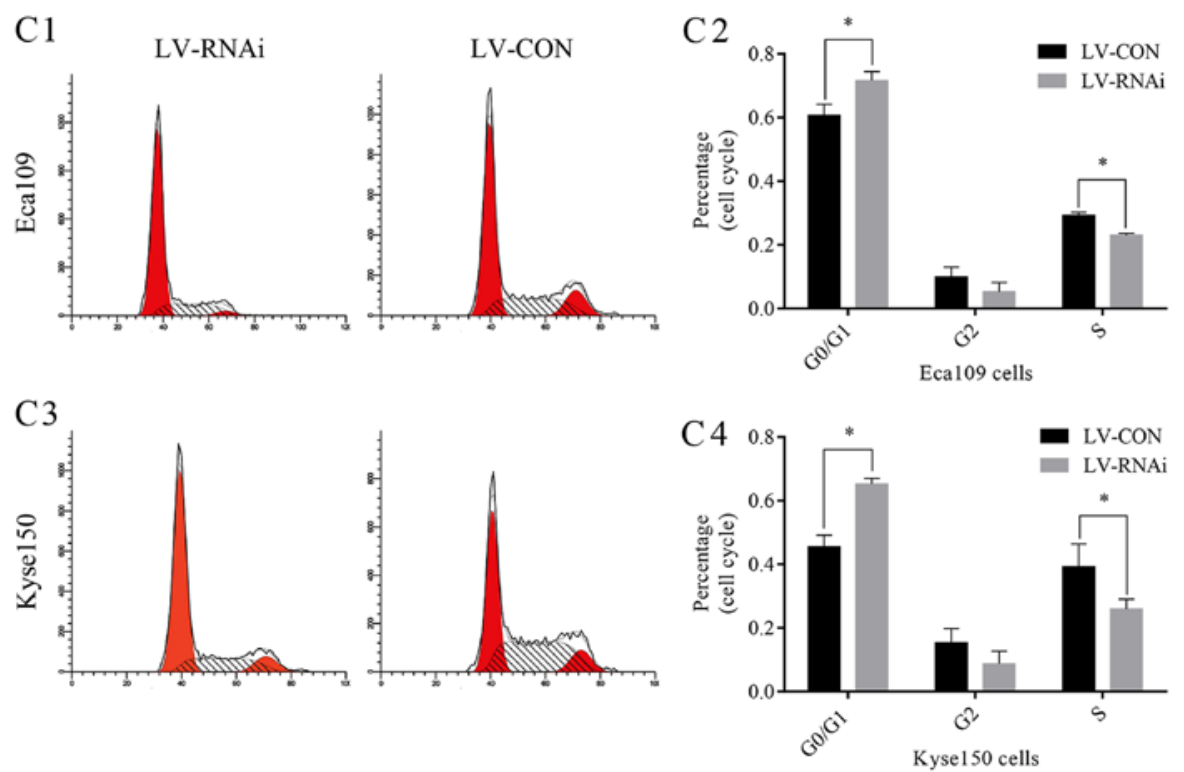

$\mathrm{D}$

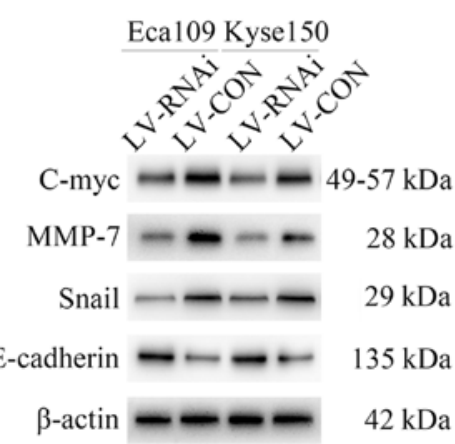

Figure 3. Continued. (C1-C4) Cell cycle was mainly arrested in the G0/G1 phase in the LV-RNAi group and the decreased proportion of cells in S phase was attributed to the cells arrested in G0/G1 phase. Three independent experiments were performed, and the results are presented as the mean \pm standard deviation; " $\mathrm{p}<0.05$. (D) TNFAIP2 regulate the expression of genes upstream and downstream of Wnt/ $\beta$-catenin. LV-RNAi-mediated TNFAIP2 knockdown increased the expression level of p-GSK-3 $\beta$ and downregulated the expression of $\beta$-catenin, which is a key factor in the Wnt/ $\beta$-catenin signaling pathway. Expression profiles of cyclin D1, c-myc, MMP-7, Snail and E-cadherin between the LV-RNAi and LV-CON group were compared. $\beta$-actin was used as a loading control.

A

$\beta$-catenin

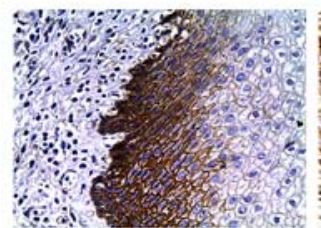

Normal

B

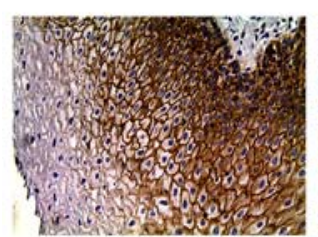

Normal

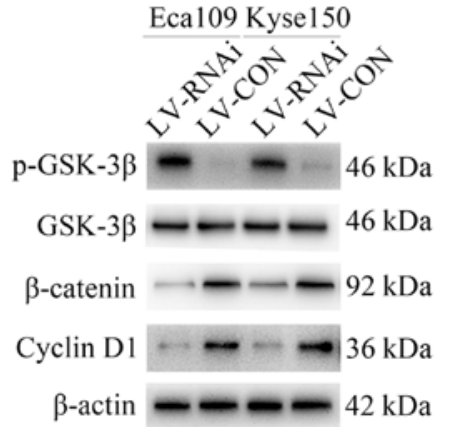

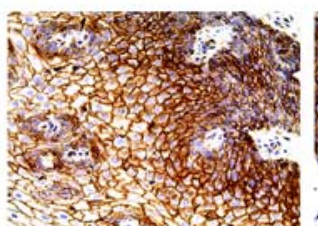

T1 stage



Tl stage

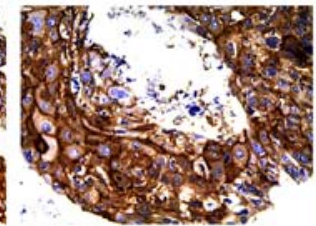

T2 stage

E-cadherin

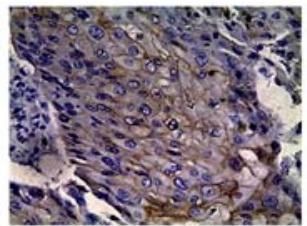

T2 stage

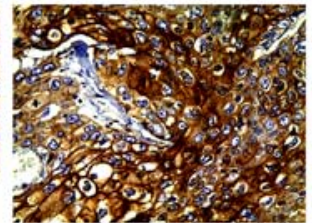

T3 stage

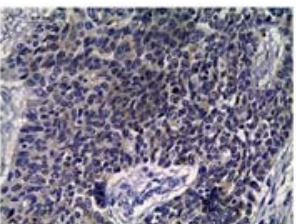

T3 stage

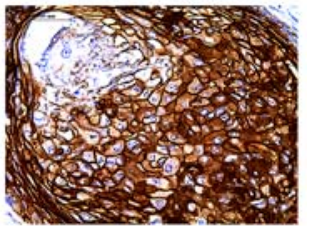

T4 stage

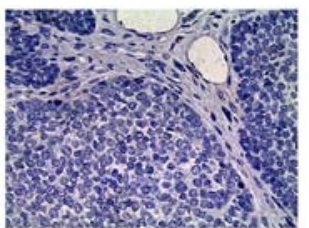

T4 stage

Figure 4. Expression of $\beta$-catenin and E-cadherin in ESCC samples. Magnification, $x$ 40. (A) $\beta$-catenin was highly expressed in membranes and the cytoplasm. (B) The higher the $\mathrm{T}$ stage, the lower expression of E-cadherin.

be upregulated in the cytoplasm of cells from tumors with an advanced $\mathrm{T}$ stage. The results of the Pearson Chi-squared test confirmed the correlations between TNFAIP2 and $\beta$-catenin expression profiles (Table I). 
Based on the western blot results of Snail and E-cadherin, we hypothesized that TNFAIP2 regulated E-cadherin expression via $\beta$-catenin. Hence, immunohistochemical staining analysis with E-cadherin antibody was conducted with 55 paraffinembedded specimens to identify correlations between the expression profiles of TNFAIP2 and E-cadherin. The results showed that low and high E-cadherin expression levels were detected in $37(67 \%)$ and 18 (33\%) samples, respectively, as compared with adjacent normal epithelium (Fig. 4B). Pearson Chi-squared test results revealed a correlation between TNFAIP2 and E-cadherin expression levels, as shown in Table I. Moreover, the Pearson Chi-squared test results showed that $\beta$-catenin and E-cadherin expression levels were correlated with $\mathrm{T}$ stage $(\mathrm{p}=0.001$ and $\mathrm{p}<0.001$, respectively) and immunohistochemical staining analysis (Fig. 4).

Based on these results, we extrapolated that TNFAIP2 operates through the $\mathrm{Wnt} / \beta$-catenin signaling pathway.

\section{Discussion}

The aim of the present study was to determine whether TNFAIP2 overexpression promotes cellular proliferation, migration and invasion via activation of the $\mathrm{Wnt} / \beta$-catenin signaling pathway in ESCC. TNFAIP2, a major response gene to the B94 protein, was found to be upregulated by $\mathrm{TNF} \alpha$ in human endothelial cells (9). The biological function of TNFAIP2 in ESCC remains vague as there is a limited number of studies detailing the mechanisms underlying the influence of TNFAIP2 expression in biological functions of tumors. Nonetheless, some of these studies state that EBV-encoded LMP1 potently induces TNFAIP2 expression via necrosis factor $-\kappa B$, which presents a possible independent prognostic indicator of the migration and invasion of nasopharyngeal carcinoma $(11,12)$. High levels of TNFAIP2 mRNA in unfractionated bone marrow and APL-treated blasts with all-trans-retinoic acid was found to result in the upregulation of TNFAIP2 (10), and the malignant Reed-Sternberg cells in classical Hodgkin's lymphoma, LP cells in nodular lymphocyte-predominant Hodgkin lymphoma, and B-cells in primary mediastinal B-cell lymphoma aberrantly express TNFAIP2 (24). Accordingly, it is essential to examine TNFAIP2 expression and to investigate its biological functions in ESCC.

TNFAIP2 may play a significant role as a potential prognostic factor and therapeutic target in ESCC, as TNFAIP2 is overexpressed in ESCC and is pivotally associated with T, N and UICC stages in ESCC patients. Moreover, lower TNFAIP2 expression is more closely related with T, N and UICC stages, as compared with higher TNFAIP2 expression, particularly in advanced T and UICC stages. Moreover, Kaplan-Meier analysis indicated that overall survival in patients with TNFAIP2 overexpression was correlated with low TNFAIP2 expression. Accordingly, TNFAIP2 may be a potential prognostic factor in ESCC and associated with tumor progression.

$\mathrm{TNF} \alpha$, as a major cytokine in the tumor microenvironment, is capable of stimulating tumor cell growth and dissemination (3). Based on the findings of the aforementioned and present studies $(9-11,24)$, TNFAIP2 was overexpressed in TNF $\alpha$-treated ESCC cell lines. Hence, we speculated that LV-RNAi-mediated TNFAIP2 knockdown could stifle cell growth, colony formation, proliferation, migration, invasion and the cell cycle of the tumorigenic ESCC cell lines Eca109 and Kyse150, which demonstrated that TNFAIP2 plays an important role in the tumor-facilitating effects of TNF $\alpha$ in ESCC. The study results also showed that the tumor-prohibitive function (cell growth, colony formation, proliferation and cell cycle) of LV-RNAi-mediated TNFAIP2 knockdown was mainly correlated with its role in arresting the cell cycle at the G0/G1 stage. LV-RNAi-mediated TNFAIP2 knockdown could downregulate cyclin D1 and c-Myc, which are closely associated with tumorigenesis (25-27). Meanwhile, LV-RNAi-mediated TNFAIP2 knockdown decreased MMP-7 and Snail expression, but increased expression of E-cadherin. To the best of our knowledge, ectopic expression of MMP-7, $\beta$-catenin and E-cadherin plays an indispensable role in facilitating invasiveness and decreasing cell-to-cell adhesion in ESCC (28).

Taking previous studies (29-31) and our data into consideration, we believe that expression levels of cyclin D1, c-Myc, MMP-7 and Snail (all of them are downstream targets of $\beta$-catenin) were mainly decreased via LV-RNAi-mediated TNFAIP2 knockdown in the ESCC cell lines. We further demonstrated that $\beta$-catenin mRNA and protein expression levels were downregulated through LV-RNAi-mediated TNFAIP2 knockdown. Meanwhile, p-GSK-3 $\beta$, which is located upstream of $\beta$-catenin, was upregulated by LV-RNAi-mediated TNFAIP2 knockdown. We hypothesized that $\beta$-catenin and E-cadherin expression levels were closely correlated with TNFAIP2 overexpression. To confirm this hypothesis, immunohistochemical staining analysis was performed to validate the suspected correlations between TNFAIP2 and $\beta$-catenin and E-cadherin. The results of the Pearson Chi-squared test immunohistochemical analysis were coincident with our hypothesis.

Although, significant findings were revealed in the present study, there were some limitations that should be addressed. For example, since there was a limited number of adequate clinical samples and limited follow-up, we only investigated whether TNFAIP2 expression is an independent risk factor for ESCC using univariate Cox regression analysis, which showed that there were no significant differences with TNFAIP2, $\beta$-catenin, E-cadherin, T, UICC and N stage, as covariates. Notwithstanding these limitations, further studies are warranted to investigate the function of TNFAIP2 in ESCC.

In summary, we identified a potential role of TNFAIP2 overexpression in ESCC as an oncogene. Therefore, TNFAIP2 overexpression facilitates proliferation and metastasis via activation of the Wnt/ $\beta$-catenin pathway in ESCC. Meanwhile, TNFAIP2 may be a potential and momentous prognostic indicator in ESCC patients.

\section{Acknowledgements}

We thank Professor Tingxiu Xiang (Chongqing Key Laboratory of Molecular Oncology and Epigenetics) and Ke Yang (Department of Pathological Center, Chongqing Medical University) for providing technical support.

\section{References}

1. Torre LA, Bray F, Siegel RL, Ferlay J, Lortet-Tieulent J and Jemal A: Global cancer statistics, 2012. CA Cancer J Clin 65: $87-108,2015$ 
2. Rustgi AK and El-Serag HB: Esophageal carcinoma. N Engl J Med 371: 2499-2509, 2014

3. Crusz SM and Balkwill FR: Inflammation and cancer: Advances and new agents. Nat Rev Clin Oncol 12: 584-596, 2015.

4. Semenzato G: Tumour necrosis factor: A cytokine with multiple biological activities. Br J Cancer 61: 354-361, 1990.

5. Locksley RM, Killeen $\mathrm{N}$ and Lenardo MJ: The TNF and TNF receptor superfamilies: Integrating mammalian biology. Cell 104: 487-501, 2001

6. Szlosarek P, Charles KA and Balkwill FR: Tumour necrosis factor-alpha as a tumour promoter. Eur J Cancer 42: 745-750, 2006.

7. Balkwill F; F. B: Tumour necrosis factor and cancer. Nat Rev Cancer 9: 361-371, 2009.

8. Sun J, Han J, Zhao Y, Zhu Q and Hu J: Curcumin induces apoptosis in tumor necrosis factor-alpha-treated HaCaT cells. Int Immunopharmacol 13: 170-174, 2012.

9. Sarma V, Wolf FW, Marks RM, Shows TB and Dixit VM: Cloning of a novel tumor necrosis factor-alpha-inducible primary response gene that is differentially expressed in development and capillary tube-like formation in vitro. J Immunol 148: 3302-3312, 1992.

10. Rusiniak ME, Yu M, Ross DT, Tolhurst EC and Slack JL: Identification of B94 (TNFAIP2) as a potential retinoic acid target gene in acute promyelocytic leukemia. Cancer Res 60 : 1824-1829, 2000.

11. Chen LC, Chen CC, Liang Y, Tsang NM, Chang YS and Hsueh C: A novel role for TNFAIP2: Its correlation with invasion and metastasis in nasopharyngeal carcinoma. Mod Pathol 24 175-184, 2011.

12. Chen CC, Liu HP, Chao M, Liang Y, Tsang NM, Huang HY, Wu CC and Chang YS: NF- $\mathrm{B}$-mediated transcriptional upregulation of TNFAIP2 by the Epstein-Barr virus oncoprotein, LMP1, promotes cell motility in nasopharyngeal carcinoma. Oncogene 33: 3648-3659, 2014.

13. Hadisaputri YE, Miyazaki T, Suzuki S, Yokobori T, Kobayashi T, Tanaka N, Inose T, Sohda $\mathrm{M}$ and Kuwano H: TNFAIP8 overexpression: Clinical relevance to esophageal squamous cell carcinoma. Ann Surg Oncol 19 (Suppl 3): S589-S596, 2012.

14. Yoshida A, Tsuta K, Ohno M, Yoshida M, Narita Y, Kawai A, Asamura $\mathrm{H}$ and Kushima R: STAT6 immunohistochemistry is helpful in the diagnosis of solitary fibrous tumors. Am J Surg Pathol 38: 552-559, 2014

15. Luo J, Zhang C, Wang C, Li L, Li C, Li Q, Zhang M and Wu Q: Miz-1 promotes the proliferation of esophageal cancer cells via suppression of p21 and release of p21-arrested cyclin D1. Oncol Rep 35: 3532-3540, 2016.

16. Budwit-Novotny DA, McCarty KS, Cox EB, Soper JT, Mutch DG, Creasman WT, Flowers JL and McCarty KS Jr: Immunohistochemical analyses of estrogen receptor in endometrial adenocarcinoma using a monoclonal antibody. Cancer Res 46: 5419-5425, 1986.

17. Smith J, Robida MD, Acosta K, Vennapusa B, Mistry A Martin G, Yates A and Hnatyszyn HJ: Quantitative and qualitative characterization of Two PD-L1 clones: SP263 and E1L3N Diagn Pathol 11: 44, 2016.
18. Kreso A, van Galen P, Pedley NM, Lima-Fernandes E, Frelin C, Davis T, Cao L, Baiazitov R, Du W, Sydorenko N, et al: Self-renewal as a therapeutic target in human colorectal cancer. Nat Med 20: 29-36, 2014.

19. Livak KJ and Schmittgen TD: Analysis of relative gene expression data using real-time quantitative PCR and the $2^{-\Delta \Delta C_{\mathrm{T}}}$ method. Methods 25: 402-408, 2001.

20. Huang Q, Whitington T, Gao P, Lindberg JF, Yang Y, Sun J, Väisänen MR, Szulkin R, Annala M, Yan J, et al: A prostate cancer susceptibility allele at 6q22 increases RFX6 expression by modulating HOXB13 chromatin binding. Nat Genet 46: 126-135, 2014.

21. Xu Y, Ma H, Yu H, Liu Z, Wang LE, Tan D, Muddasani R, Lu V, Ajani JA, Wang Y, et al: The miR-184 binding-site rs8126 T>C polymorphism in TNFAIP2 is associated with risk of gastric cancer. PLoS One 8: e64973, 2013.

22. Zhang J, Yu H, Zhang Y, Zhang X, Zheng G, Gao Y, Wang C and Zhou L: A functional TNFAIP2 3'-UTR rs8126 genetic polymorphism contributes to risk of esophageal squamous cell carcinoma. PLoS One 9: e109318, 2014.

23. Liu Z, Wei S, Ma H, Zhao M, Myers JN, Weber RS, Sturgis EM and Wei Q: A functional variant at the miR-184 binding site in TNFAIP2 and risk of squamous cell carcinoma of the head and neck. Carcinogenesis 32: 1668-1674, 2011.

24. Kondratiev S, Duraisamy S, Unitt CL, Green MR, Pinkus GS, Shipp MA, Kutok JL, Drapkin RI and Rodig SJ: Aberrant expression of the dendritic cell marker TNFAIP2 by the malignant cells of Hodgkin lymphoma and primary mediastinal large B-cell lymphoma distinguishes these tumor types from morphologically and phenotypically similar lymphomas. Am J Surg Pathol 35: 1531-1539, 2011.

25. Wang Y, Zhou X, Zhu H, Liu S, Zhou C, Zhang G, Xue L, Lu N, Quan L, Bai J, et al: Overexpression of EB1 in human esophageal squamous cell carcinoma (ESCC) may promote cellular growth by activating beta-catenin/TCF pathway. Oncogene 24: 6637-6645, 2005

26. Gao Y, Song C, Hui L, Li CY, Wang J, Tian Y, Han X, Chen Y, Tian DL, Qiu X, et al: Overexpression of RNF146 in non-small cell lung cancer enhances proliferation and invasion of tumors through the Wnt/ $\beta$-catenin signaling pathway. PLoS One 9: e85377, 2014

27. Qin YR, Tang H, Xie F, Liu H, Zhu Y, Ai J, Chen L, Li Y, Kwong DL, Fu L, et al: Characterization of tumor-suppressive function of SOX6 in human esophageal squamous cell carcinoma. Clin Cancer Res 17: 46-55, 2011

28. Shimada Y and Sato F: Molecular factors related to metastasis of esophageal squamous cell carcinoma. Esophagus 4: 7-18, 2007.

29. Qiu HB, Zhang LY, Ren C, Zeng ZL, Wu WJ, Luo HY, Zhou ZW and $\mathrm{Xu}$ RH: Targeting $\mathrm{CDH} 17$ suppresses tumor progression in gastric cancer by downregulating Wnt/ $\beta$-catenin signaling. PLoS One 8: e56959, 2013.

30. Stewart DJ: Wnt signaling pathway in non-small cell lung cancer. J Natl Cancer Inst 106: djt356, 2014.

31. Moon RT: Wnt/beta-catenin pathway. Sci STKE 2005: $\mathrm{cm} 1-\mathrm{cm} 1$, 2005. 